\title{
Release of Cornell 607-612: Common Bean Breeding Lines with Resistance to White Mold
}

\author{
Phillip D. Griffiths ${ }^{1}$ \\ Department of Horticulture, Cornell University NYSAES, 315 Hedrick Hall, \\ Geneva, NY 14456
}

\section{Eric Sandsted and Donald Halseth \\ Department of Horticulture, Cornell University, Ithaca, NY 14853}

Additional index words. Phaseolus vulgaris L., Sclerotinia sclerotiorum, dry bean

The New York State Agricultural Experiment Station announces the release of six dry bean (Phaseolus vulgaris L.) breeding lines with resistance to white mold [Sclerotinia sclerotiorum (Lib.) de Bary]. These breeding lines, 'Cornell 607' dark red kidney (DRK), 'Cornell 608' DRK, 'Cornell 609' black bean (BB), 'Cornell 610' BB, 'Cornell 611' light red kidney (LRK), and Cornell 612 LRK, represent germplasm with high levels of resistance to white mold developed through greenhouse selection and evaluation in multistate greenhouse and field comparisons through the W-2150 regional project.

White mold is an important disease of common bean worldwide reducing both yield and quality of seed harvested. Plant protection from white mold can be conferred through both plant architecture and physiological resistance (Kolkman and Kelly, 2003; Schwartz et al., 1978). Physiological resistance can be selected using several approaches for the selection of breeding lines (Hunter et al., 1981; Schwartz et al., 1987; Steadman et al., 1997). A technique known as the "straw test," which shows a very high correlation with field resistance, was used in the selection of these materials (Petzoldt and Dickson, 1996; Singh and Teran, 2008; Teran and Singh, 2009). This test was used to develop the six breeding lines by inoculating two petioles on each plant in the populations as described previously (Griffiths et al., 2004; Teran et al., 2006).

\section{Origin and Description}

Lines 'Cornell 607', 'Cornell 608', 'Cornell 609', 'Cornell 610', 'Cornell 611', and 'Cornell 612 ' were selected from three lineages of recurrent selection using previously developed breeding lines as sources of resistance to white mold (Griffiths, 2009). 'Cornell 607' (DRK) and 'Cornell 608' (DRK) selections were developed from a cross between 'Cornell 603' (Griffiths, 2009) and the DRK cultivar Cabernet (Seminis Vegetable Seeds, Woodland,

Received for publication 27 Mar. 2012. Accepted for publication 16 May 2012.

${ }^{1}$ To whom reprint requests should be addressed; e-mailpdg8@cornell.edu. a 1 to 5 scale, where $1=$ no progression of symptoms beyond the first node, $2=$ some progression of symptoms beyond the first node, 3 = progression of symptoms to the second node, $4=$ progression of symptoms beyond the second node, and $5=$ complete susceptibility and death of the plant.

Selection was undertaken for four generations $\left(\mathrm{F}_{3}\right.$ to $\left.\mathrm{F}_{7}\right)$ using single seed selection to advance the most promising lines. The advanced lines were tested in replicated greenhouse screens at the $F_{8}$ generation, and the two lines showing the highest level of resistance to white mold within the DRK lineages based on their reactions to infection using the straw test approach were 'Cornell 607' and 'Cornell 608'. The remaining breeding lines were developed using the same approach. BB lines 'Cornell 609' and 'Cornell 610 ' were developed as $\mathrm{F}_{8}$ replicated trial selections from lineages selected through recurrent selection and single-seed descent from a cross between 'Cornell 604' (Griffiths, 2009) and BB cultivar T-39 (University of California Davis, Davis, CA). LRK lines 'Cornell 611' and 'Cornell 612' were also selected using this approach following the generation of breeding lineages from a cross between 'Cornell 605' (Griffiths, 2009) and 'Wallace' (Cornell University, Ithaca, NY).

The six dry bean (Phaseolus vulgaris L.) breeding lines being released represent three market classes, LRK and DRK in the Andean type, and BB in the Mesoamerican type (Fig. 1). The breeding lines have been evaluated for white mold resistance in replicated greenhouse trials with multistate entries for the $2010 \mathrm{~W}-2150$ multistate regional project (Table 1). All six breeding lines performed well using the modified rating scale of 1 to 9 (Teran et al., 2006) scoring between 2.44 and 4.40 based on a mean of 20 inoculated plants for each entry and with scores in the same range or better than the resistant checks 'G-122' and 'A-195'.

Field evaluations of the breeding lines were made in replicated trials in Livingstone County, NY, and Tompkins County, NY, in

Fig. 1. Dry seed of 'Cornell 607', 'Cornell 608', 'Cornell 609', 'Cornell 610', 'Cornell 611', and 'Cornell 612 ' relative to commercial cultivars Montcalm, T-39, and Cabernet in similar market classes of common bean.

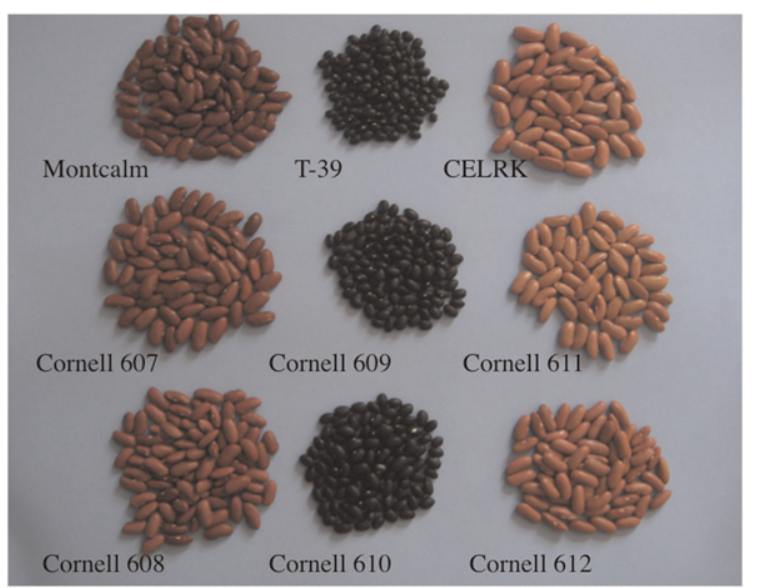

CA). $F_{1}$ hybrid seeds generated from the six crosses were increased to generate segregating were advanced to create $40 \mathrm{~F}_{3}$ sister lines from each of the six populations from single $F_{2}$ These were sown for inoculation Greenhouse inoculations for the development of the white mold-resistant breeding 2- to 3-d-old actively growing mycelia of white field in Murray, Orleans County, NY (He Sparks, MD). Plants were grown in $15 \mathrm{~cm} \times$ (1) two $3-\mathrm{cm}$ straws placed on petioles cut $5 \mathrm{~cm}$ selections of resistant plants were made based on aggressiveness and severity of the white mold growth. Ratings were made based on

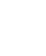

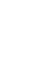

.


Table 1. Greenhouse evaluation of 'Cornell 607', '608', '609', '610', '611', and '612' in 2010 greenhouse trials at Cornell University, NY.

\begin{tabular}{lccc}
\hline Entry & $\begin{array}{c}\text { No. of } \\
\text { plants rated }\end{array}$ & $\begin{array}{c}\text { Mean } \\
\text { disease rating }\end{array}$ & Rank \\
\hline Cornell 607 & 10 & $2.44 \mathrm{i}^{\mathrm{y}}$ & 1 \\
Cornell 611 & 10 & $2.50 \mathrm{i}$ & 2 \\
Cornell 605 & 10 & $3.00 \mathrm{hi}$ & 3 \\
VCW54 & 10 & $3.10 \mathrm{~g}-\mathrm{i}$ & 4 \\
Cornell 610 & 9 & $3.11 \mathrm{~g}-\mathrm{i}$ & 5 \\
Cornell 612 & 9 & $3.22 \mathrm{f}-\mathrm{i}$ & 6 \\
G122 & 10 & $3.90 \mathrm{f}-\mathrm{h}$ & 7 \\
C08709 & 8 & $4.00 \mathrm{f}-\mathrm{h}$ & 8 \\
Cornell 609 & 10 & $4.10 \mathrm{f}-\mathrm{h}$ & 9 \\
Cornell 608 & 10 & $4.40 \mathrm{e}-\mathrm{g}$ & 10 \\
A195 & 10 & $4.50 \mathrm{ef}$ & 11 \\
ND080547 & 10 & $5.60 \mathrm{de}$ & 12 \\
NE2-09-16 & 10 & $5.80 \mathrm{~d}$ & 13 \\
P07751 & 10 & $6.20 \mathrm{~cd}$ & 14 \\
NE2-06-8 & 10 & $6.40 \mathrm{~cd}$ & 15 \\
NE2-09-4 & 10 & $7.20 \mathrm{bc}$ & 16 \\
NE2-09-19 & 10 & $7.30 \mathrm{bc}$ & 17 \\
P07863 & 10 & $7.30 \mathrm{bc}$ & 17 \\
Beryl & 10 & $7.90 \mathrm{ab}$ & 19 \\
37-2 & 10 & $8.20 \mathrm{ab}$ & 20 \\
NE2-09-1 & 10 & $8.30 \mathrm{ab}$ & 21 \\
ND060514 & 10 & $8.40 \mathrm{ab}$ & 22 \\
NE1-09-20 & 10 & $8.50 \mathrm{ab}$ & 23 \\
NE2-09-6 & 10 & $8.50 \mathrm{ab}$ & 23 \\
50-2 & 10 & $8.80 \mathrm{a}$ & 25 \\
NE2-09-14 & 8 & $8.88 \mathrm{a}$ & 26 \\
NE2-09-10 & 9 & $8.89 \mathrm{a}$ & 27 \\
NE2-09-12 & 10 & $8.90 \mathrm{a}$ & 28 \\
70-1 & 10 & $9.00 \mathrm{a}$ & 29 \\
Ex-Rico (Bunsi) & 10 & $9.00 \mathrm{a}$ & 29 \\
Hystyle & 10 & $9.00 \mathrm{a}$ & 29 \\
\hline Table & $10 p$ & 10 &
\end{tabular}

${ }^{2}$ Table represents relative ranking and means grouping differences when compared with the resistant control G-122 and susceptible checks ExRico and Beryl. Results based on data submitted to W-2150 greenhouse trials (2011).

${ }^{y}$ Means separation according to Duncan's multiple range test $(P \leq 0.05)$.

2010 (Tables 2 to 4). The DRK lines 'Cornell 607 ' and 'Cornell 608' yielded $3025.2 \mathrm{~kg} \cdot \mathrm{ha}^{-1}$ and $2929.2 \mathrm{~kg} \cdot \mathrm{ha}^{-1}$ in the Livingstone County trial and $3903.2 \mathrm{~kg} \cdot \mathrm{ha}^{-1}$ and $3412.8 \mathrm{~kg} \cdot \mathrm{ha}^{-1}$ in the Tompkins County trial, respectively (Table 2). Both lines had seed sizes and maturities in line with commercial DRK cultivars being grown. The BB releases 'Cornell 609' and 'Cornell 610' yielded higher in the Livingstone County trial generating $2508.1 \mathrm{~kg} \cdot \mathrm{ha}^{-1}$ and $2423.3 \mathrm{~kg} \cdot \mathrm{ha}^{-1}$, respectively; however, the performance for yield in the Tompkins County trial was low compared with commercial BB cultivars yielding only $2540.5 \mathrm{~kg} \cdot \mathrm{ha}^{-1}$ and $2969.9 \mathrm{~kg} \cdot \mathrm{ha}^{-1}$, respectively (Table 3 ). The LRK releases 'Cornell 611' and 'Cornell 612' yielded $3077.1 \mathrm{~kg} \cdot \mathrm{ha}^{-1}$ and $3102.2 \mathrm{~kg} \cdot \mathrm{ha}^{-1}$, respectively, in the Livingstone County trial, and $3536.6 \mathrm{~kg} \cdot \mathrm{ha}^{-1}$ and $4009.1 \mathrm{~kg} \cdot \mathrm{ha}^{-1}$, respectively, in the Tompkins County trial (Table 4). The LRK lines show improvement for both seed size and maturity over 'Cornell 605' (Griffiths, 2009), which was the donor parent for white mold resistance in these selections. These new releases represent new germplasm that can be used for developing commercial cultivars of dry bean with enhanced resistance to white mold in the LRK, DRK, and BB market classes.

Table 2. Field-testing of dark red kidney breeding lines 'Cornell 607' and 'Cornell 608' in Livingston County, NY, and Tompkins County, NY, 2010.

\begin{tabular}{|c|c|c|c|c|c|}
\hline \multicolumn{6}{|c|}{ Dark red kidney trial, Livingston County, NY, plant date 21 June } \\
\hline Entry & Source & Yield $\left(\mathrm{kg} \cdot \mathrm{ha}^{-1}\right)$ & $\begin{array}{c}\text { Harvest } \\
\text { index (\%) }\end{array}$ & $\begin{array}{l}\text { Seed size } \\
\text { (seeds/lb) }\end{array}$ & $\begin{array}{c}\text { Days to } 90 \% \\
\text { dry pods }\end{array}$ \\
\hline K06012 & Mich. St. & $3413.3 \mathrm{a}^{\mathrm{z}}$ & $50.8 \mathrm{a}$ & 1968.7 a & $90.0 \mathrm{~b}$ \\
\hline Cornell 607 & Cornell & $3025.2 \mathrm{ab}$ & $50.9 \mathrm{a}$ & $1840.8 \mathrm{bc}$ & $94.3 \mathrm{a}$ \\
\hline Cornell 608 & Cornell & $2929.9 \mathrm{a}-\mathrm{c}$ & $45.3 \mathrm{ab}$ & $1863.5 \mathrm{~b}$ & $96.0 \mathrm{a}$ \\
\hline Montcalm & Mich St. & $2880.2 \mathrm{a}-\mathrm{c}$ & $43.6 \mathrm{ab}$ & $1745.4 \mathrm{c}$ & $98.0 \mathrm{a}$ \\
\hline Cornell 603 & Cornell & $2843.3 \mathrm{a}-\mathrm{c}$ & $43.4 \mathrm{ab}$ & $1879.0 \mathrm{ab}$ & $95.3 \mathrm{a}$ \\
\hline GTS104 & Gentec & $2462.2 \mathrm{bc}$ & $40.4 \mathrm{~b}$ & $1782.0 \mathrm{bc}$ & $96.7 \mathrm{a}$ \\
\hline Cabernet & Seminis & $2108.0 \mathrm{c}$ & $41.0 \mathrm{~b}$ & $1856.9 \mathrm{~b}$ & $94.7 \mathrm{a}$ \\
\hline
\end{tabular}

Dark red kidney trial, Tompkins County, NY, plant date 18 June

\begin{tabular}{llcccc}
\hline Entry & Source & Yield $\left(\mathrm{kg} \cdot \mathrm{ha}^{-1}\right)$ & $\begin{array}{c}\text { Harvest } \\
\text { index }(\%)\end{array}$ & $\begin{array}{c}\text { Seed size } \\
(\text { seeds/lb) }\end{array}$ & $\begin{array}{c}\text { Days to } 90 \% \\
\text { dry pods }\end{array}$ \\
\hline GTS 104 & Gentec & $4076.3 \mathrm{a}$ & $45.5 \mathrm{bc}$ & $1637.6 \mathrm{~d}$ & $98.3 \mathrm{a}$ \\
GTS 106 & Gentec & $3903.4 \mathrm{ab}$ & $46.5 \mathrm{bc}$ & $1686.5 \mathrm{~cd}$ & $95.8 \mathrm{~b}$ \\
Cornell 607 & Cornell & $3903.2 \mathrm{ab}$ & $53.9 \mathrm{a}$ & $1753.8 \mathrm{bc}$ & $98.8 \mathrm{a}$ \\
K08228 & Mich St. & $3900.9 \mathrm{ab}$ & $44.7 \mathrm{c}$ & $1819.9 \mathrm{ab}$ & $93.5 \mathrm{c}$ \\
Cabernet & Seminis & $3779.9 \mathrm{ab}$ & $46.0 \mathrm{bc}$ & $1683.0 \mathrm{~d}$ & $94.3 \mathrm{bc}$ \\
K06012 & Mich St. & $3719.3 \mathrm{a}-\mathrm{c}$ & $47.4 \mathrm{~b}$ & $1861.7 \mathrm{a}$ & $93.0 \mathrm{c}$ \\
Montcalm & Mich St. & $3642.0 \mathrm{~b}-\mathrm{d}$ & $37.9 \mathrm{~d}$ & $1604.9 \mathrm{~d}$ & $99.0 \mathrm{a}$ \\
Cornell 608 & Cornell & $3412.8 \mathrm{~cd}$ & $45.2 \mathrm{c}$ & $1786.4 \mathrm{ab}$ & $99.0 \mathrm{a}$ \\
Cornell 603 & Cornell & $3319.2 \mathrm{~d}$ & $46.1 \mathrm{bc}$ & $1866.9 \mathrm{ab}$ & $93.3 \mathrm{c}$ \\
\hline
\end{tabular}

${ }^{2}$ Means separation according to Duncan's multiple range test $(P \leq 0.05)$.

Table 3. Field-testing of black bean breeding lines 'Cornell 609' and 'Cornell 610' in Livingston County, NY, and Tompkins County, NY, 2010.

\begin{tabular}{|c|c|c|c|c|c|}
\hline \multicolumn{6}{|c|}{ Black bean trial, Livingston County, NY, plant date 21 June } \\
\hline Entry & Source & Yield $\left(\mathrm{kg} \cdot \mathrm{ha}^{-1}\right)$ & $\begin{array}{c}\text { Harvest } \\
\text { index }(\%)\end{array}$ & $\begin{array}{c}\text { Seed size } \\
\text { (seeds } / \mathrm{kg} \text { ) }\end{array}$ & $\begin{array}{l}\text { Days to } 90 \% \\
\text { dry pods }\end{array}$ \\
\hline GTS 1103 & Gentec & $3190.3 \mathrm{a}^{\mathrm{z}}$ & $48.7 \mathrm{a}$ & $4566.4 \mathrm{~cd}$ & $93.3 \mathrm{~d}$ \\
\hline Black Velvet & Seminis & $3145.4 \mathrm{a}$ & $42.8 \mathrm{ab}$ & $4151.3 \mathrm{e}$ & $97.0 \mathrm{~b}$ \\
\hline Zorro & Mich. St. & $2823.4 \mathrm{ab}$ & $46.1 \mathrm{a}$ & $4639.1 \mathrm{~b}-\mathrm{d}$ & $93.3 \mathrm{~d}$ \\
\hline Cornell 609 & Cornell & $2508.1 \mathrm{a}-\mathrm{c}$ & $42.7 \mathrm{ab}$ & $3622.8 \mathrm{f}$ & $111.3 \mathrm{a}$ \\
\hline Cornell 610 & Cornell & $2423.3 \mathrm{a}-\mathrm{c}$ & $44.0 \mathrm{ab}$ & $3552.9 \mathrm{f}$ & $110.3 \mathrm{a}$ \\
\hline Eclipse & Coop NRS & $2338.1 \mathrm{a}-\mathrm{c}$ & $38.8 \mathrm{ab}$ & $5137.4 \mathrm{a}$ & $90.0 \mathrm{e}$ \\
\hline $\mathrm{T}-39$ & ADM & $2311.5 \mathrm{a}-\mathrm{c}$ & $42.1 \mathrm{ab}$ & $4929.5 \mathrm{ab}$ & $95.7 \mathrm{~b}-\mathrm{d}$ \\
\hline Shania & $\mathrm{ADM}$ & $2309.3 \mathrm{a}-\mathrm{c}$ & $38.4 \mathrm{ab}$ & $5099.2 \mathrm{a}$ & $96.3 \mathrm{bc}$ \\
\hline Midnight & IBS & $2254.6 \mathrm{a}-\mathrm{c}$ & $38.8 \mathrm{ab}$ & $4981.7 \mathrm{~b}-\mathrm{d}$ & $95.0 \mathrm{~cd}$ \\
\hline Jaguar & $\mathrm{ADM}$ & $2254.4 \mathrm{a}-\mathrm{c}$ & $38.9 \mathrm{ab}$ & $4979.3 \mathrm{ab}$ & $94.0 \mathrm{~b}-\mathrm{d}$ \\
\hline $96-148$ & Cornell & $2253.2 \mathrm{a}-\mathrm{c}$ & $39.7 \mathrm{ab}$ & $4894.8 \mathrm{ab}$ & $95.0 \mathrm{~cd}$ \\
\hline Condor & $\mathrm{ADM}$ & $2202.0 \mathrm{a}-\mathrm{c}$ & $41.1 \mathrm{ab}$ & $4475.3 \mathrm{~d}$ & $96.0 \mathrm{~b}-\mathrm{d}$ \\
\hline Jet Black & Idaho BS & $2133.3 \mathrm{a}-\mathrm{c}$ & $39.0 \mathrm{ab}$ & $4813.3 \mathrm{a}-\mathrm{d}$ & $95.0 \mathrm{~b}-\mathrm{d}$ \\
\hline Jlly Roger & Idaho BS & $1765.0 \mathrm{bc}$ & $38.4 \mathrm{ab}$ & $4720.0 \mathrm{~b}-\mathrm{d}$ & $93.0 \mathrm{~b}-\mathrm{d}$ \\
\hline
\end{tabular}

Black bean trial, Tompkins County, NY, plant date 18 June

\begin{tabular}{llcccc}
\hline Entry & Source & Yield $\left(\mathrm{kg} \cdot \mathrm{ha}^{-1}\right)$ & $\begin{array}{c}\text { Harves } \\
\text { index }(\%)\end{array}$ & $\begin{array}{c}\text { Seed size } \\
(\text { seeds } / \mathrm{kg})\end{array}$ & $\begin{array}{c}\text { Days to } 90 \% \\
\text { dry pods }\end{array}$ \\
\hline Zorro & Mich. St. & $4874.9 \mathrm{a}$ & $56.2 \mathrm{bc}$ & $4251.1 \mathrm{c}-\mathrm{e}$ & $97.0 \mathrm{de}$ \\
Jet Black & Idaho BS & $4861.7 \mathrm{a}$ & $58.5 \mathrm{ab}$ & $4402.1 \mathrm{bc}$ & $96.8 \mathrm{de}$ \\
COB 223-99 & Gentec & $4720.2 \mathrm{ab}$ & $57.8 \mathrm{~b}$ & $4491.3 \mathrm{ab}$ & $92.5 \mathrm{~h}$ \\
GTS 1103 & Gentec & $4681.0 \mathrm{a}-\mathrm{c}$ & $56.9 \mathrm{~b}$ & $4359.6 \mathrm{~b}-\mathrm{d}$ & $92.8 \mathrm{gh}$ \\
COB2159-00 & Gentec & $4679.5 \mathrm{a}-\mathrm{c}$ & $60.3 \mathrm{a}$ & $4044.3 \mathrm{f}$ & $93.0 \mathrm{gh}$ \\
Shania & ADM & $4605.0 \mathrm{a}-\mathrm{d}$ & $50.1 \mathrm{fg}$ & $4477.5 \mathrm{~b}$ & $96.5 \mathrm{e}$ \\
Midnight & Cornell & $4602.2 \mathrm{a}-\mathrm{d}$ & $48.7 \mathrm{hg}$ & $4520.5 \mathrm{ab}$ & $102.3 \mathrm{~b}$ \\
Condor & Mich St. & $4526.3 \mathrm{a}-\mathrm{d}$ & $51.8 \mathrm{ef}$ & $4379.4 \mathrm{~b}-\mathrm{d}$ & $94.8 \mathrm{f}$ \\
96-148 & Cornell & $4486.8 \mathrm{a}-\mathrm{d}$ & $52.9 \mathrm{de}$ & $4097.9 \mathrm{ef}$ & $100.3 \mathrm{c}$ \\
Jolly Roger & Idaho BS & $4438.6 \mathrm{~b}-\mathrm{d}$ & $56.0 \mathrm{bc}$ & $4164.3 \mathrm{ef}$ & $95.8 \mathrm{ef}$ \\
Eclipse & NDSU & $4404.9 \mathrm{~b}-\mathrm{d}$ & $51.6 \mathrm{ef}$ & $4488.1 \mathrm{ab}$ & $94.3 \mathrm{fg}$ \\
Black Velvet & Seminis & $4370.0 \mathrm{~b}-\mathrm{d}$ & $43.4 \mathrm{i}$ & $4013.5 \mathrm{f}$ & $100.8 \mathrm{bc}$ \\
Jaguar & Mich St. & $4357.3 \mathrm{~b}-\mathrm{d}$ & $54.3 \mathrm{~cd}$ & $4647.3 \mathrm{a}$ & $91.8 \mathrm{~h}$ \\
T-39 & UC Davis & $4306.6 \mathrm{~cd}$ & $50.5 \mathrm{fg}$ & $4236.8 \mathrm{de}$ & $98.3 \mathrm{~d}$ \\
Cornell 610 & Cornell & $2969.9 \mathrm{e}$ & $43.2 \mathrm{i}$ & $3337.8 \mathrm{~h}$ & $105.8 \mathrm{a}$ \\
Cornell 609 & Cornell & $2540.5 \mathrm{~d}$ & $41.4 \mathrm{~h}$ & $3730.8 \mathrm{ef}$ & $105.3 \mathrm{bc}$ \\
\hline
\end{tabular}

${ }^{\mathrm{z}}$ Means separation according to Duncan's multiple range test $(P \leq 0.05)$.

\section{Availability}

Small amounts of seed are available from P.D. Griffiths, New York State Agricultural
Experiment Station, Cornell University, Department of Horticultural Sciences, 314 Hedrick Hall, Geneva, NY 14456 with a material transfer agreement. 
Table 4. Field-testing of light red kidney breeding lines 'Cornell 611' and 'Cornell 612' in Livingston County, NY, and Tompkins County, NY, 2010.

\begin{tabular}{|c|c|c|c|c|c|}
\hline \multicolumn{6}{|c|}{ Light red kidney trial, Livingston County, NY, plant date 21 June } \\
\hline Entry & Source & Yield $\left(\mathrm{kg} \cdot \mathrm{ha}^{-1}\right)$ & $\begin{array}{c}\text { Harvest } \\
\text { index }(\%)\end{array}$ & $\begin{array}{l}\text { Seed size } \\
(\text { seeds/kg) }\end{array}$ & $\begin{array}{c}\text { Days to } 90 \% \\
\text { dry pods }\end{array}$ \\
\hline NY105 & Cornell & $4143.0 \mathrm{a}^{\mathrm{z}}$ & $59.3 \mathrm{a}$ & 1547.0 ef & $88.0 \mathrm{de}$ \\
\hline A-47 & Cornell & $3818.0 \mathrm{ab}$ & $54.4 \mathrm{bc}$ & $1462.3 \mathrm{f}$ & $88.7 \mathrm{c}-\mathrm{e}$ \\
\hline CELRK & UC Davis & $3556.5 \mathrm{a}-\mathrm{c}$ & $56.5 \mathrm{ab}$ & 1538.8 ef & $86.3 \mathrm{e}$ \\
\hline NY104 & Cornell & $3481.7 \mathrm{a}-\mathrm{c}$ & $52.5 \mathrm{~b}-\mathrm{e}$ & $1592.4 \mathrm{~d}-\mathrm{f}$ & $88.3 \mathrm{de}$ \\
\hline Wallace & Cornell & $3468.2 \mathrm{a}-\mathrm{c}$ & $51.6 \mathrm{c}-\mathrm{e}$ & $2232.6 \mathrm{a}$ & $89.7 \mathrm{c}-\mathrm{e}$ \\
\hline A-36 & Cornell & $3442.1 \mathrm{~b}-\mathrm{d}$ & $53.8 \mathrm{~b}-\mathrm{d}$ & $1677.0 \mathrm{c}-\mathrm{e}$ & $89.0 \mathrm{c}-\mathrm{e}$ \\
\hline A-41 & Cornell & $3342.7 b-d$ & $48.8 \mathrm{e}-\mathrm{g}$ & $1948.2 \mathrm{~b}$ & $91.0 \mathrm{c}-\mathrm{e}$ \\
\hline Pink Panther & Seminis & $3231.7 b-d$ & $50.6 \mathrm{c}-\mathrm{f}$ & 1544.8 ef & $91.7 \mathrm{~cd}$ \\
\hline Cornell 612 & Cornell & $3102.2 \mathrm{~cd}$ & 46. $\mathrm{fg}$ & $1650.6 \mathrm{c}-\mathrm{e}$ & $93.3 \mathrm{c}$ \\
\hline Cornell 611 & Cornell & $3077.1 \mathrm{~cd}$ & $49.7 \mathrm{~d}-\mathrm{g}$ & 1538.8 ef & $92.0 \mathrm{~cd}$ \\
\hline A-45 & Cornell & $3061.4 \mathrm{~cd}$ & $45.7 \mathrm{~g}$ & $1702.0 \mathrm{~cd}$ & $92.7 \mathrm{~cd}$ \\
\hline A-38 & Cornell & $2978.0 \mathrm{~cd}$ & $46.3 \mathrm{fg}$ & $1887.1 \mathrm{~b}$ & $92.7 \mathrm{~cd}$ \\
\hline Cornell 605 & Cornell & $2922.8 \mathrm{~cd}$ & 47. $\mathrm{e}-\mathrm{g}$ & $1737.9 \mathrm{c}$ & $102.0 \mathrm{~b}$ \\
\hline RedKanner & Cornell & $2745.0 \mathrm{~d}$ & $46.0 \mathrm{fg}$ & $2011.3 \mathrm{~b}$ & $106.3 \mathrm{a}$ \\
\hline \multicolumn{6}{|c|}{ Light red kidney, Tompkins County, NY, plant date 18 June } \\
\hline Entry & Source & Yield $\left(\mathrm{kg} \cdot \mathrm{ha}^{-1}\right)$ & $\begin{array}{c}\text { Harvest } \\
\text { index }(\%)\end{array}$ & $\begin{array}{c}\text { Seed size } \\
(\text { seeds/kg) }\end{array}$ & $\begin{array}{c}\text { Days to } 90 \% \\
\text { dry pods }\end{array}$ \\
\hline$\overline{A-47}$ & Cornell & $4819.7 \mathrm{a}$ & $51.0 \mathrm{~b}$ & $1374.6 \mathrm{i}$ & 100.0 ef \\
\hline RedKanner & Cornell & $4761.7 \mathrm{a}$ & $51.6 \mathrm{~b}$ & $1735.0 \mathrm{c}$ & $107.0 \mathrm{a}$ \\
\hline NY105 & Cornell & $4666.4 \mathrm{ab}$ & $55.2 \mathrm{a}$ & $1475.5 \mathrm{gh}$ & $94.8 \mathrm{k}$ \\
\hline NY118 & Cornell & $4663.5 \mathrm{ab}$ & $47.3 \mathrm{~cd}$ & $1613.8 \mathrm{~d}$ & $104.5 \mathrm{~b}$ \\
\hline A-36 & Cornell & $4553.2 \mathrm{a}-\mathrm{c}$ & $51.4 \mathrm{~b}$ & $1606.1 \mathrm{de}$ & 95.3 jk \\
\hline Wallace & Cornell & $4404.7 \mathrm{a}-\mathrm{d}$ & $52.9 \mathrm{ab}$ & $2092.2 \mathrm{a}$ & $98.5 \mathrm{f}-\mathrm{h}$ \\
\hline NY104 & Cornell & $4390.7 b-d$ & $47.6 \mathrm{~cd}$ & $1567.0 \mathrm{~d}-\mathrm{f}$ & $98.0 \mathrm{gh}$ \\
\hline COB1966-99 & Gentec & $4353.9 \mathrm{~b}-\mathrm{e}$ & $37.4 \mathrm{~g}$ & 1416.0 hi & $103.5 \mathrm{bc}$ \\
\hline CPB2092-99 & Gentec & $4325.4 \mathrm{~b}-\mathrm{f}$ & $40.9 \mathrm{f}$ & $1416.0 \mathrm{hi}$ & $102.8 \mathrm{bc}$ \\
\hline Pink Panther & Seminis & $4242.4 \mathrm{c}-\mathrm{f}$ & $42.1 \mathrm{ef}$ & $1478.8 \mathrm{gh}$ & $96.8 \mathrm{~h}-\mathrm{j}$ \\
\hline A-41 & Cornell & $4132.3 \mathrm{~d}-\mathrm{h}$ & $46.2 \mathrm{~cd}$ & $1885.6 \mathrm{~b}$ & $99.0 \mathrm{e}-\mathrm{g}$ \\
\hline Cornell 612 & Cornell & $4009.1 \mathrm{e}-\mathrm{h}$ & $44.9 \mathrm{c}-\mathrm{e}$ & $1578.1 \mathrm{de}$ & $99.0 \mathrm{e}-\mathrm{g}$ \\
\hline KO6619 & Mich St. & $3993.4 \mathrm{f}-\mathrm{h}$ & $39.4 \mathrm{fg}$ & $1637.0 \mathrm{~d}$ & $100.8 \mathrm{de}^{\circ}$ \\
\hline A-38 & Cornell & $3982.7 \mathrm{f}-\mathrm{h}$ & $44.6 \mathrm{de}$ & $1642.4 \mathrm{~d}$ & $95.8 \mathrm{i}-\mathrm{k}$ \\
\hline Cornell 605 & Cornell & $3978.5 \mathrm{f}-\mathrm{h}$ & $50.7 \mathrm{~b}$ & $1623.7 \mathrm{~d}$ & $103.3 \mathrm{bc}$ \\
\hline CELRK & UC Davis & $3940.3 \mathrm{gh}$ & $47.8 \mathrm{c}$ & $1531.8 \mathrm{e}-\mathrm{g}$ & 92.51 \\
\hline Chinook2000 & Mich St. & $3881.3 \mathrm{gh}$ & $40.0 \mathrm{f}$ & $1732.4 \mathrm{c}$ & $102.0 \mathrm{~cd}$ \\
\hline$A-45$ & Cornell & $3858.4 \mathrm{hi}$ & $40.4 \mathrm{f}$ & $1571.9 \mathrm{~d}-\mathrm{f}$ & $97.3 \mathrm{~g}-\mathrm{i}$ \\
\hline 1062-V98 & Cornell & $3798.0 \mathrm{hi}$ & $41.6 \mathrm{f}$ & $1610.5 \mathrm{~d}$ & $100.8 \mathrm{de}$ \\
\hline Cornell 611 & Cornell & $3536.6 \mathrm{i}$ & $45.4 \mathrm{~cd}$ & $1499.1 \mathrm{fg}$ & $97.8 \mathrm{gh}$ \\
\hline
\end{tabular}

${ }^{\mathrm{z}}$ Means separation according to Duncan's multiple range test $(P \leq 0.05)$.

\section{Literature Cited}

Boodley, J.W. and R. Sheldrake, Jr. 1982. Cornell peat-lite mixes for commercial plant growing. New York Agr. Exp. Sta. Agr. Info. Bul. 43.

Griffiths, P.D. 2009. Release of Cornell 601-606: Common bean breeding lines with resistance to white mold. HortScience 44:463-465.

Griffiths, P.D., M.M. Jahn, and M.H. Dickson. 2004. Cornell 501: A white mold tolerant snap bean breeding line. HortScience 39:1507-1508.

Hunter, J.E., M.H. Dickson, and J.A. Cigna. 1981. Limited-term inoculation: A method to screen bean plants for partial resistance to white mold. Plant Dis. 65:414-417.

Kolkman, J.M. and J.D. Kelly. 2003. QTL conferring resistance and avoidance to white mold in common bean. Crop Sci. 43:539-548.

Petzoldt, R. and M.H. Dickson. 1996. Straw test for resistance to white mold in beans. Annu. Rep. Bean Improv. Coop. 39:142-143.

Schwartz, H.F., D.H. Casciano, J.A. Asenga, and D.R. Wood. 1987. Field measurement of white mold effects upon dry beans with genetic resistance or upright architecture. Crop Sci. 27:699-702.

Schwartz, H.F., J.R. Steadman, and D.P. Coyne. 1978. Influence of Phaseolus vulgaris blossoming characteristics and canopy structure upon reaction to Sclerotinia sclerotiorum. Phytopathology 68:465-470.

Singh, S. and H. Teran. 2008. Evolution of screening methods for identification of physiological resistance to white mold in dry bean. Annu. Rep. Bean Improv. Coop. 51:40-41.

Steadman, J.R., K. Powers, and B. Higgens. 1997. Screening common bean for white mold resistance using detached leaves. Annu. Rep. Bean Improv. Coop. 40:140-141.

Teran, H., M. Lema, H.F. Schwartz, R. Duncan, R. Gilbertson, and S. Singh. 2006. Modified Pedzoldt and Dickson scale for white mold rating of common bean. Annu. Rep. Bean Improv. Coop. 49:115-116.

Teran, H. and S.P. Singh. 2009. Efficacy of three greenhouse screening methods for the identification of physiological resistance to white mold in dry beans. Can. J. Plant Sci. 89:755-762. 